\title{
Construction of Bivariate Copulas on a Multivariate Exponentially Weighted Moving Average Control Chart
}

\author{
Sirasak Sasiwannapong ${ }^{1}$, Saowanit Sukparungsee ${ }^{1, *}$, Piyapatr Busababodhin ${ }^{2}$, Yupaporn Areepong ${ }^{1}$ \\ ${ }^{1}$ Department of Applied Statistics, Faculty of Applied Science, King Mongkut's University of Technology North Bangkok, Thailand \\ ${ }^{2}$ Department of Mathematics, Faculty of Science, Mahasarakham University, Thailand
}

Received April 13, 2020 ; Revised June 24, 2020; Accepted July 10, 2020

\section{Cite This Paper in the following Citation Styles}

(a): [1] Sirasak Sasiwannapong, Saowanit Sukparungsee, Piyapatr Busababodhin, Yupaporn Areepong , "Construction of Bivariate Copulas on a Multivariate Exponentially Weighted Moving Average Control Chart," Mathematics and Statistics, Vol. 8, No. 5, pp. 520 - 526, 2020. DOI: 10.13189/ms.2020.080504.

(b): Sirasak Sasiwannapong, Saowanit Sukparungsee, Piyapatr Busababodhin, Yupaporn Areepong (2020). Construction of Bivariate Copulas on a Multivariate Exponentially Weighted Moving Average Control Chart. Mathematics and Statistics, 8(5), 520 - 526. DOI: 10.13189/ms.2020.080504.

Copyright $\mathrm{C} 2020$ by authors, all rights reserved. Authors agree that this article remains permanently open access under the terms of the Creative Commons Attribution License 4.0 International License

\begin{abstract}
The control chart is an important tool in multivariate statistical process control (MSPC), which for monitoring, control, and improvement of the process control. In this paper, we propose six types of copula combinations for use on a Multivariate Exponentially Weighted Moving Average (MEWMA) control chart. Observations from an exponential distribution with dependence measured with Kendall's tau for moderate and strong positive and negative dependence (where $\tau= \pm 0.5, \pm 0.8)$ among the observations were generated by using Monte Carlo simulations to measure the Average Run Length (ARL) as the performance metric and should be sufficiently large when the process is in-control on a MEWMA control chart. In this study, we develop an approach performance on the MEWMA control chart based on copula combinations by using the Monte Carlo simulations. The results show that the out-of-control $\left(\mathrm{ARL}_{1}\right)$ values for $\boldsymbol{\lambda}=\mathbf{0 . 0 5}$ were less than for $\boldsymbol{\lambda}=\mathbf{0 . 1 0}$ in almost all cases. The performances of the Farlie-Gumbel-Morgenstern $\times$ Ali-Mikhail-Haq copula combination was superior to the others for all shifts with strong positive dependence among the observations and $\boldsymbol{\lambda}=\mathbf{0 . 0 5}$. Moreover, when the magnitudes of the shift were very large, the performance metric values for observations with moderate and strong positive and negative dependence followed the same pattern.
\end{abstract}

Keywords Marginal, Joint Distribution, Multivariate Control Chart, Monte Carlo Simulation

\section{Introduction}

Multivariate Statistical Process Control (MSPC) is an important method for process monitoring, control and improvement in many areas such as engineering, economics, environmental statistics, finance and etc. For example, in automotive production quality control depends on correlated variables such as the lifetimes of the components in the engine, etc. A control chart is a common tool for MSPC for detecting changes in the vector means of the process. Multivariate control charts are generalizations of their univariate counterparts [1]. Hotelling's $T^{2}$ was the first multivariate control chart [2], followed by the Multivariate Exponentially Weighted Moving Average (MEWMA) control chart as a better alternative for detecting small shifts in the process vector mean $[3,4]$. Most multivariate detection procedures are based on the assumption that the observations are independent and identically distributed (i.i.d.) and follow a multivariate normal distribution. However, many processes are non-normal and correlated, so multivariate control charts need to be able to cope with related joint distributions. Hence, Kuvattana et al. [5] and Sukparungsee et al. [6] introduced the copula to address this requirement.

Copulas are functions that join multivariate distributions to their one-dimensional marginal distribution functions in which the one-dimensional margins are uniform on the interval $(0,1)[7]$. They are used to explain the dependence between random variables and are based on a 
representation of Sklar's theorem [8]. A new way of constructing asymmetric copulas was introduced by Mukherjee et al. [9], and later on copulas have been applied to MSPC [10]. Several other studies have proposed and compared the performance of bivariate copulas on the multivariate control charts [11-14]. Herein, we present the efficiency of the combinations of bivariate copulas constructed for shifts in the process vector mean on a MEWMA control chart when observations follow an exponential distribution.

\section{Research Methodology}

This paper is organized into the following sections: in section 2.1 the multivariate exponentially weighted moving average (MEWMA) control chart. Section 2.2, we review copulas function and constructing bivariate copulas. Section 2.3 describes the dependence measure of data and finally section 2.4 provides the ARL and the simulation study.

\subsection{The Multivariate Exponentially Weighted Moving Average (MEWMA) Control Chart}

The MEWMA control chart was first developed by Lowry et al. [4]. The given observations $W_{1}, W_{2}, \rightleftharpoons$ from a d-variate Gaussian distribution $N(\boldsymbol{\mu}, \boldsymbol{\Sigma})$, for $\mathrm{i}=1,2, \ldots$, can be defined as

$$
\mathbf{Z}_{i}=\Lambda \mathbf{W}_{i}+(1-\Lambda) \mathbf{Z}_{i-1}
$$

where $\mathbf{Z}_{i}$ is a vector of variable values from the data and

$\Lambda$ is a diagonal matrix with entries $\lambda_{1}, \lambda_{2}, \vec{\sim}, \lambda_{d}$, for $0 \leq \lambda \leq 1$ and $\lambda_{1}=\lambda_{2}=\stackrel{\vec{\nu}}{=} \boldsymbol{\lambda}_{d}=\boldsymbol{\lambda}$.

The quantity plotted on the control chart is

$$
T_{i}^{2}=\mathbf{Z}_{i}^{\prime} \Sigma_{i}^{-1} \mathbf{Z}_{i},
$$

where $\Sigma_{i}=\frac{\lambda}{2-\lambda}\left[1-(1-\lambda)^{2 i}\right] \Sigma$.

When $\boldsymbol{\lambda}_{1}=\boldsymbol{\lambda}_{2}=\underset{\boldsymbol{\nu}}{\boldsymbol{\nu}}=\boldsymbol{\lambda}_{\boldsymbol{d}}=\boldsymbol{\lambda}$ on the interval $(0,1)$ (as assumed in this study), the control chart signals a shift in the mean vector when $T_{i}^{2}>H$ where $H$ is the control limit chosen for the desired in-control process. Generally, the Average Run Length (ARL) can be used to measure the performance of a MEWMA control chart. It depends on the degree of dependence between the variables measured using the covariance matrix $\sum$ and the scalar-weighted $\lambda$ associated with the past observations. We consider a bivariate EWMA control chart and the control limit $H$ for the in-control process $\mathrm{ARL}_{0}=370$.

\subsection{Copulas Function and Constructing Bivariate Copulas}

Theoretically, for the copula function according to Sklar's theorem [8] for a bivariate case, let $X$ and $Y$ be continuous variables with joint distribution function $G$ and marginal cumulative distributions $F(x)$ and $F(y)$, respectively. Consequently, $G(x, y)=C(F(x), F(y) ; \theta)$ with copula $C:[0,1]^{2} \rightarrow[0,1]$ where $\theta$ is a parameter of the copula. Theoretically, let $A$ and $B$ be bivariate copulas. It follows that $C_{\alpha \beta}(u, v)=A\left(u^{\alpha}, v^{\beta}\right) B\left(u^{1-\alpha}, v^{1-\beta}\right)$, where $C_{\alpha, \beta}$ is a copula with parameters $\alpha, \boldsymbol{\beta} \in \mathbf{I}$ and $\boldsymbol{\alpha} \neq \boldsymbol{\beta}$ [15]. If $\boldsymbol{\alpha}=\boldsymbol{\beta}=\mathbf{1}$, then $C_{1,1}=A$, and if $\boldsymbol{\alpha}=\boldsymbol{\beta}=\mathbf{0}$ then $C_{0,0}=B$. Similarly, if $C_{(u, v)} \neq C_{(v, u)}$ we have an asymmetric copula.

In accordance with Khoudraji's device [16], let $C$ be symmetric copula $C \neq \Pi_{2}$, where $\Pi_{2}$ is independence copula. A family of asymmetric copulas $C_{\alpha, \beta}$ with parameters $\mathbf{0}<\boldsymbol{\alpha}, \boldsymbol{\beta}<\mathbf{1}, \boldsymbol{\alpha} \neq \boldsymbol{\beta}$ that includes $C$ as a limiting case is given by

$$
C_{\alpha, \beta}(u, v)=u^{\alpha} v^{\beta} \cdot C\left(u^{1-\alpha}, v^{1-\beta}\right) .
$$

\subsection{Dependence Measure of the Data}

Generally, a copula can be used in the study of the dependence of association between random variables by Kendall's tau, which we implemented in this study (Table-1). Let $X$ and $Y$ be continuous random variables with copula $C$, then Kendall's tau is given by

$$
\tau=4 \iint_{\mathbf{r}^{2}} C(u, v) d C(u, v)-1 .
$$

Table 1. Kendall's tau of copula function

\begin{tabular}{|c|c|c|c|}
\hline Copula & Type & Kendall's tau & Parameter space of $\theta$ \\
\hline Clayton & Asymmetric & $\theta /(\theta / 2)$ & {$[-1, \infty) \backslash\{0\}$} \\
\hline Frank & Asymmetric & $\mathbf{A}\left(\frac{1}{\theta} \int_{0}^{\theta} \frac{t}{\boldsymbol{e}^{t}-1} d t-1\right)$ & $(-\infty, \infty) \backslash\{0\}$ \\
\hline FGM & Symmetric & $2 \theta /(3-\theta)$ & {$[-1,1]$} \\
\hline AMH & Symmetric & $\arcsin (\theta) /\left(\frac{\pi}{2}\right)$ & {$[-1,1]$} \\
\hline
\end{tabular}




\subsection{The ARL and the Simulation Study}

Theoretically, the ARL is an average number of points that must be plotted before the out-of-control condition occurs. ARL is classified into $\mathrm{ARL}_{0}$ and $\mathrm{ARL}_{1}$. $\mathrm{ARL}_{0}$ is the average number of observations before the first out-of-control point, while $\mathrm{ARL}_{1}$ is the average number of observations when the process is out-of-control. The expectations of $\mathrm{ARL}_{0}$ and $\mathrm{ARL}_{1}$ can be respectively expressed as

$$
\begin{array}{ll}
A R L_{0} \approx E_{\omega}(\kappa) \geq K ; & \text { for } \omega=\infty \\
A R L_{1} \approx E_{\omega}(\kappa \mid \kappa \geq 1) ; & \text { for } \boldsymbol{\omega}=1
\end{array}
$$

where $\boldsymbol{\omega}$ is the change point time, $\boldsymbol{\kappa}$ is the stopping time, and $E_{\omega}($.$) is the expectation under the assumption that$ the change point occurs at $\boldsymbol{\omega}$.

We ran a Monte Carlo simulation using $\mathrm{R}$ statistical software [17-20] with the 50,000 rounds and a sample size of 6,000 . The observations were generated from a copula based on an exponential distribution with mean $=1$ (for the in-control process) and shifts at level $0.01,0.05,0.1,0.5,1$, and 5 (for the out-of-control process). The performance of the MEWMA control chart was assessed for $\lambda=0.05$ and 0.10 . For all combinations of copulas, setting $\boldsymbol{\omega}$ corresponds to Kendall's tau for moderate and strong positive and negative dependence $(\tau= \pm 0.5, \pm-0.8)$.

\section{Results}

The simulation results are reported in Tables 2 to 9, in which the results are only empirical. The aim of the study was to optimize the parameters for constructing bivariate copulas $(\boldsymbol{\alpha}, \boldsymbol{\beta})$, as shown in Equation (3), for which we used the Maximum pseudo-likelihood estimator method [21]. For the in-control process on the MEWMA control chart, the desired $\mathrm{ARL}_{0}=370$ was set for each copula combination. The results in Tables 2 and 3 indicate moderate positive dependence among the observations ( $\boldsymbol{\tau}=\mathbf{0 . 5}$ ), Tables 4 and 5 strong positive dependence ( $\tau=\mathbf{0 . 8}$ ), Tables 6 and 7 moderate negative dependence $(\tau=-0.5)$, and Tables 8 to 9 show strong negative dependence $(\tau=-\mathbf{0 . 8})$.

\begin{tabular}{|c|c|c|c|c|c|c|}
\hline \multirow{2}{*}{ Shift } & \multicolumn{6}{|c|}{ Copula combinations } \\
\hline & {$[1]$} & {$[2]$} & {$[3]$} & {$[4]$} & {$[5]$} & {$[6]$} \\
\hline 0.01 & 329.14 & 330.20 & 329.25 & 332.24 & 332.61 & 329.24 \\
\hline 0.05 & 236.15 & 240.22 & 233.78 & 242.38 & 234.52 & 241.08 \\
\hline 0.10 & 194.51 & 197.77 & 194.46 & 200.24 & 197.11 & 199.02 \\
\hline 0.50 & 12.74 & 14.41 & 13.39 & 10.27 & 12.87 & 10.48 \\
\hline 1.00 & 1.74 & 1.92 & 1.81 & 2.10 & 1.70 & 2.19 \\
\hline 5.00 & 1.02 & 1.02 & 1.09 & 1.14 & 1.07 & 1.03 \\
\hline UCL & 10.69 & 12.24 & 11.21 & 14.03 & 10.66 & 15.30 \\
\hline$\alpha$ & 0.566 & 0.858 & 0.953 & 0.855 & 0.161 & 0.045 \\
\hline$\beta$ & 0.617 & 0.466 & 0.906 & 0.841 & 0.128 & 0.032 \\
\hline
\end{tabular}

Table 2. $\mathrm{ARL}_{1}$ of the MEWMA control chart with moderate positive dependence $(\boldsymbol{\tau}=0.5, \boldsymbol{\lambda}=0.05)$

Note that: Copula combinations i.e.
[1] Clayton $\times$ FGM
[2] Clayton $\times$ Frank
[3] Clayton $\times \mathrm{AMH}$
[4] FGM $\times$ Frank
[5] $\mathrm{FGM} \times \mathrm{AMH}$
[6] Frank $\times \mathrm{AMH}$

\begin{tabular}{|c|c|c|c|c|c|c|}
\hline \multirow{2}{*}{ Shift } & \multicolumn{6}{|c|}{ Copula combinations } \\
\hline & {$[1]$} & {$[2]$} & {$[3]$} & {$[4]$} & {$[5]$} & {$[6]$} \\
\hline 0.01 & 330.39 & 332.88 & 332.15 & 334.11 & 333.3 & 332.28 \\
\hline 0.05 & 243.41 & 246.95 & 245.29 & 248.99 & 242.75 & 251.73 \\
\hline 0.10 & 204.81 & 209.86 & 137.03 & 211.93 & 208.05 & 211.82 \\
\hline 0.50 & 15.30 & 16.52 & 20.87 & 17.30 & 15.69 & 11.86 \\
\hline 1.00 & 2.06 & 2.27 & 2.16 & 2.43 & 2.03 & 2.52 \\
\hline 5.00 & 1.02 & 1.03 & 1.01 & 1.04 & 1.09 & 1.20 \\
\hline UCL & 13.69 & 15.56 & 14.26 & 17.77 & 13.73 & 19.35 \\
\hline$\alpha$ & 0.566 & 0.858 & 0.953 & 0.855 & 0.161 & 0.045 \\
\hline$\beta$ & 0.617 & 0.466 & 0.906 & 0.841 & 0.128 & 0.032 \\
\hline
\end{tabular}

Table 3. $\mathrm{ARL}_{1}$ of the MEWMA control chart with moderate positive dependence $(\boldsymbol{\tau}=0.5, \lambda=0.10)$

Note that: Copula combinations i.e.
[1] Clayton $\times$ FGM
[2] Clayton $\times$ Frank
[3] Clayton $\times \mathrm{AMH}$
[4] FGM $\times$ Frank
[5] $\mathrm{FGM} \times \mathrm{AMH}$
[6] Frank $\times$ AMH 
Table 4. $\mathrm{ARL}_{1}$ of the MEWMA control chart with strong positive dependence ( $\boldsymbol{\tau}=0.8, \boldsymbol{\lambda}=0.05$ )

\begin{tabular}{|c|c|c|c|c|c|c|}
\hline \multirow{2}{*}{ Shift } & \multicolumn{6}{|c|}{ Copula combinations } \\
\hline & {$[1]$} & {$[2]$} & [3] & [4] & [5] & [6] \\
\hline 0.01 & 329.43 & 331.64 & 328.91 & 334.11 & 326.23 & 331.25 \\
\hline 0.05 & 237.85 & 243.71 & 238.09 & 244.72 & 210.57 & 243.13 \\
\hline 0.10 & 194.65 & 203.84 & 196.25 & 205.23 & 126.87 & 202.94 \\
\hline 0.50 & 15.03 & 16.48 & 14.07 & 17.48 & 7.84 & 11.83 \\
\hline 1.00 & 2.01 & 2.36 & 1.93 & 2.47 & 1.67 & 2.47 \\
\hline 5.00 & 1.03 & 1.07 & 1.10 & 1.10 & 1.02 & 1.04 \\
\hline UCL & 13.11 & 17.84 & 11.99 & 20.52 & 10.24 & 20.70 \\
\hline$\alpha$ & 0.457 & 0.567 & 0.635 & 0.95 & 0.405 & 0.069 \\
\hline$\beta$ & 0.457 & 0.779 & 0.652 & 0.949 & 0.676 & 0.007 \\
\hline
\end{tabular}

Note that: Copula combinations i.e.

[1] Clayton $\times$ FGM [2] Clayton $\times$ Frank [3] Clayton $\times$ AMH [4] FGM $\times$ Frank [5] FGM $\times$ AMH [6] Frank $\times$ AMH

Table 5. $\mathrm{ARL}_{1}$ of the MEWMA control chart with strong positive dependence ( $\boldsymbol{\tau}=0.8, \boldsymbol{\lambda}=0.10$ )

\begin{tabular}{|c|c|c|c|c|c|c|}
\hline \multirow{2}{*}{ Shift } & \multicolumn{6}{|c|}{ Copula combinations } \\
\hline & [1] & {$[2]$} & [3] & [4] & [5] & [6] \\
\hline 0.01 & 331.30 & 330.08 & 333.33 & 335.59 & 330.10 & 336.65 \\
\hline 0.05 & 246.74 & 249.45 & 246.12 & 256.45 & 240.26 & 232.98 \\
\hline 0.10 & 207.94 & 211.95 & 139.75 & 217.58 & 203.87 & 152.70 \\
\hline 0.50 & 16.52 & 18.31 & 10.68 & 19.68 & 15.17 & 13.33 \\
\hline 1.00 & 2.37 & 2.67 & 2.24 & 2.87 & 2.01 & 2.88 \\
\hline 5.00 & 1.04 & 1.08 & 1.13 & 1.12 & 1.00 & 1.32 \\
\hline UCL & 16.57 & 22.50 & 15.15 & 26.31 & 13.22 & 26.65 \\
\hline$\alpha$ & 0.457 & 0.567 & 0.635 & 0.95 & 0.405 & 0.069 \\
\hline$\beta$ & 0.457 & 0.779 & 0.652 & 0.949 & 0.676 & 0.007 \\
\hline
\end{tabular}

Note that: Copula combinations i.e.

[1] Clayton $\times$ FGM [2] Clayton $\times$ Frank [3] Clayton $\times$ AMH [4] FGM $\times$ Frank [5] FGM $\times$ AMH [6] Frank $\times$ AMH

Table 6. $\mathrm{ARL}_{1}$ of the MEWMA control chart with moderate negative dependence ( $\boldsymbol{\tau}=-0.5, \boldsymbol{\lambda}=0.05$ )

\begin{tabular}{|c|c|c|c|c|c|c|}
\hline \multirow{2}{*}{ Shift } & \multicolumn{6}{|c|}{ Copula combinations } \\
\hline & [1] & {$[2]$} & [3] & {$[4]$} & [5] & {$[6]$} \\
\hline 0.01 & 324.71 & 328.54 & 326.98 & 325.05 & 330.92 & 328.38 \\
\hline 0.05 & 232.22 & 235.22 & 235.14 & 233.45 & 235.47 & 235.28 \\
\hline 0.10 & 192.74 & 192.98 & 191.74 & 191.16 & 193.04 & 193.90 \\
\hline 0.50 & 14.47 & 16.13 & 14.30 & 15.92 & 13.97 & 16.06 \\
\hline 1.00 & 1.85 & 2.45 & 1.82 & 2.36 & 1.80 & 2.29 \\
\hline 5.00 & 1.02 & 1.02 & 1.02 & 1.02 & 1.02 & 1.02 \\
\hline $\mathrm{UCL}$ & 11.32 & 14.53 & 11.1 & 13.95 & 10.97 & 13.40 \\
\hline$\alpha$ & 0.982 & 0.999 & 0.99 & 0.919 & 1.000 & 0.149 \\
\hline$\beta$ & 0.999 & 0.999 & 0.998 & 1.000 & 0.915 & 0.029 \\
\hline
\end{tabular}

Note that: Copula combinations i.e.
[1] Clayton $\times$ FGM
[2] Clayton $\times$ Frank
[3] Clayton $\times \mathrm{AMH}$
[4] FGM $\times$ Frank [5] FGM $\times$ AMH [6] Frank $\times$ AMH 
Table 7. $\mathrm{ARL}_{1}$ of the MEWMA control chart with moderate negative dependence ( $\boldsymbol{\tau}=-0.5, \lambda=0.10$ )

\begin{tabular}{|c|c|c|c|c|c|c|}
\hline \multirow{2}{*}{ Shift } & \multicolumn{6}{|c|}{ Copula combinations } \\
\hline & {$[1]$} & {$[2]$} & [3] & [4] & [5] & [6] \\
\hline 0.01 & 328.63 & 323.78 & 326.92 & 330.17 & 326.36 & 326.79 \\
\hline 0.05 & 233.24 & 228.14 & 233.79 & 231.26 & 233.64 & 227.13 \\
\hline 0.10 & 191.54 & 185.83 & 190.78 & 187.44 & 191.02 & 186.32 \\
\hline 0.50 & 16.39 & 16.20 & 16.10 & 16.32 & 16.11 & 16.27 \\
\hline 1.00 & 2.26 & 2.80 & 2.22 & 2.73 & 2.19 & 2.65 \\
\hline 5.00 & 1.02 & 1.03 & 1.02 & 1.03 & 1.02 & 1.02 \\
\hline UCL & 14.25 & 17.68 & 14.00 & 17.14 & 13.86 & 16.38 \\
\hline$\alpha$ & 0.982 & 0.999 & 0.990 & 0.919 & 1.000 & 0.149 \\
\hline$\beta$ & 0.999 & 0.999 & 0.998 & 1.000 & 0.915 & 0.029 \\
\hline
\end{tabular}

Note that: Copula combinations i.e.

[1] Clayton $\times$ FGM [2] Clayton $\times$ Frank [3] Clayton $\times$ AMH [4] FGM $\times$ Frank [5] FGM $\times$ AMH [6] Frank $\times$ AMH

Table 8. $\mathrm{ARL}_{1}$ of the MEWMA control chart copulas with strong negative dependence ( $\tau=-0.8, \lambda=0.05$ )

\begin{tabular}{|c|c|c|c|c|c|c|}
\hline \multirow{2}{*}{ Shift } & \multicolumn{6}{|c|}{ Copula combinations } \\
\hline & [1] & {$[2]$} & [3] & [4] & [5] & [6] \\
\hline 0.01 & 326.37 & 328.95 & 327.01 & 326.35 & 326.71 & 322.98 \\
\hline 0.05 & 234.28 & 232.98 & 232.22 & 235.52 & 235.31 & 233.37 \\
\hline 0.10 & 192.17 & 191.68 & 194.22 & 192.31 & 194.65 & 191.90 \\
\hline 0.50 & 14.44 & 16.25 & 14.31 & 15.92 & 14.28 & 15.89 \\
\hline 1.00 & 1.84 & 2.58 & 1.82 & 2.50 & 1.82 & 2.42 \\
\hline 5.00 & 1.02 & 1.01 & 1.01 & 1.01 & 1.02 & 1.01 \\
\hline UCL & 11.32 & 15.53 & 11.09 & 14.95 & 11.12 & 14.25 \\
\hline$\alpha$ & 0.996 & 1.000 & 0.995 & 0.806 & 0.999 & 0.261 \\
\hline$\beta$ & 0.987 & 0.858 & 0.998 & 0.999 & 0.999 & 0.001 \\
\hline
\end{tabular}

Note that: Copula combinations i.e.

[1] Clayton $\times$ FGM [2] Clayton $\times$ Frank [3] Clayton $\times$ AMH $\quad$ [4] FGM $\times$ Frank [5] FGM $\times$ AMH [6] Frank $\times$ AMH

Table 9. $\mathrm{ARL}_{1}$ of the MEWMA control chart with strong negative dependence $(\boldsymbol{\tau}=-0.8, \lambda=0.10)$

\begin{tabular}{|c|c|c|c|c|c|c|}
\hline \multirow{2}{*}{ Shift } & \multicolumn{6}{|c|}{ Copula combinations } \\
\hline & [1] & {$[2]$} & [3] & {$[4]$} & {$[5]$} & {$[6]$} \\
\hline 0.01 & 326.44 & 327.60 & 325.84 & 324.42 & 328.11 & 327.16 \\
\hline 0.05 & 234.44 & 226.75 & 232.27 & 228.30 & 235.30 & 229.35 \\
\hline 0.10 & 193.59 & 187.43 & 190.09 & 186.49 & 192.91 & 185.96 \\
\hline 0.50 & 16.35 & 15.83 & 16.02 & 15.91 & 16.34 & 15.99 \\
\hline 1.00 & 2.26 & 2.88 & 2.22 & 2.82 & 2.22 & 2.75 \\
\hline 5.00 & 1.02 & 1.01 & 1.02 & 1.01 & 1.02 & 1.01 \\
\hline UCL & 14.25 & 18.77 & 13.98 & 18.12 & 14.05 & 17.40 \\
\hline$\alpha$ & 0.996 & 1.000 & 0.995 & 0.806 & 0.999 & 0.261 \\
\hline$\beta$ & 0.987 & 0.858 & 0.998 & 0.999 & 0.999 & 0.001 \\
\hline
\end{tabular}

Note that: Copula combinations i.e.
[1] Clayton $\times$ FGM
[2] Clayton $\times$ Frank
[3] Clayton $\times \mathrm{AMH}$
[4] FGM $\times$ Frank [5] FGM $\times$ AMH [6] Frank $\times$ AMH 
The results in Tables 2 to 9 show that the $\mathrm{ARL}_{1}$ values for $\boldsymbol{\lambda}=\mathbf{0 . 0 5}$ were less than those for $\boldsymbol{\lambda}=\mathbf{0 . 1 0}$ in almost all cases. The results in Tables 2 and 3 indicate that the Clayton $\times$ Ali-Mikhail-Haq (AMH) copula combination was superior to the others in almost all cases. Meanwhile, with strong positive dependence $(\tau=0.8)$ and $\lambda=0.05$, Farlie-Gumbel-Morgenstern (FGM) $\times$ AMH attained the minimum $\mathrm{ARL}_{1}$ with all shifts (Table 4). Meanwhile, for moderate negative dependence $(\tau=-0.5)$, Clayton $X$ FGM attained the minimum $\mathrm{ARL}_{1}$ with shift values at 0.01 and 0.05 (Table 6). For the results for strong negative dependence $(\boldsymbol{\tau}=\mathbf{- 0 . 8})$ and $\boldsymbol{\lambda}=\mathbf{0 . 0 5}$ (Table 8), the performance of FGM $\times$ AMH was superior to the others with shift values at 0.5 and 1 . However, when the magnitude of the shift was large $(\delta \geq 5)$, the performances of all of the copula combinations for moderate and strong positive and negative dependence were the same.

\section{Conclusions}

In this study, we investigated closed-form approximations of the ARL for MEWMA control charts using bivariate copulas constructed via Khoudraji's device, and we used Monte Carlo simulation when the marginal of the variables was exponential with $\boldsymbol{\mu}=\mathbf{l}$. The simulation results suggest that there were no meaningful differences between the performances of the bivariate copulas at a very large shift $(\delta \geq 5)$ when the observations had moderate and strong positive and negative dependence. In addition, the performances of the constructed bivariate copulas were superior to a single copula [5] for a moderate shift in a process on a MEWMA control chart. For further research, we could use the real data to compare the simulation results.

\section{Acknowledgements}

The authors are grateful to the Ministry of Science and Technology, Thailand, and the Graduate College, King Mongkut's University of Technology, North Bangkok, Thailand, for financially supporting this study. This research was funded by Office the Higher Education Commission-National Research University with contract no. KMUTNB-NRU-59-11. Finally, this research was inspired by the manuscript, "Construction of bivariate asymmetric copulas" [9]. Moreover, we would like to thank Professor Dr. Jeong-Soo Park for the idea proposed in this paper.

\section{REFERENCES}

[1] M. A. Mahmoud, P. E. Maravelakis. The performance of multivariate CUSUM control charts with estimated parameters, Journal of Statistical Computation and Simulation, Vol.83, No.4, 721-738, 2013.

[2] H. Hotelling. Multivariate Quality Control Illustrated by Air Testing of Sample Bombsights, In: Eisenhart, C., Hastay, M.W. and Wallis, W.A., Eds., Techniques of Statistical Analysis, McGraw Hill, New York, 111-184. 1947.

[3] H. Midi, A. Shabbak. Robust multivariate control chart to detect small shifts in mean, Mathematical Problems in Engineering, Vol.2011, 1-19, 2011.

[4] C. A. Lowry, W. H. Woodall, C. W. Champ, S. E. Rigdon. A multivariate exponentially weighted moving average control chart, Technometrics, Vol.34, No.1, 46-53, 1992.

[5] S. Kuvattana, S. Sukparungsee, P. Busbabodhin, Y. Areepong. Bivariate copulas on the exponentially weighted moving average control chart, Songklanakarin Journal of Science and Technology, Vol.38, No.5, 569-574, 2016.

[6] S. Sukparungsee, S. Kuvattana, P. Busbabodhin, Y. Areepong. Bivariate copulas on the Hotelling's $T^{2}$ control chart, Communications in Statistics-Simulation and Computation, Vol.47, No.2, 413-419, 2018.

[7] R. B. Nelson. An introduction to copulas, $2^{\text {nd }}$ ed, Springer, New York, 2006.

[8] A. Sklar. Random variables, Joint distribution function and copulas, Kybernetica, Vol.9, 449-460, 1973.

[9] S. Mukherjee, Y. S. Lee, J. M. Kim, J. Jang, J. S. Park. Construction of bivariate asymmetric copulas, Communications for Statistical Applications and Methods, Vol.25, No.2, 217-234, 2018.

[10] P. Busababodhin, P. Amphanthong. Copula modelling for multivariate statistical process control: a review, Communications for Statistical Applications and Methods, Vol.23, No.6, 497-515, 2016.

[11] S. Tiengket, S. Sukparungsee, P. Busababodhin, Y. Areepong. Construction of bivariate copulas on the Hotelling's $T^{2}$ control chart, Thailand Statistician, Vol.18, No.1, 1-15, 2020.

[12] S. Sasiwannapong, S. Sukparungsee, P. Busababodhin, Y. Areepong. The efficiency of constructed bivariate copulas for MEWMA and Hotelling's $T^{2}$ control charts, Communications in Statistics-Simulation and Computation, Online 25 Nov 2019, doi: 10.1080/03610918.2019.1687719

[13] S. Kuvattana, S. Sukparungsee, P. Busbabodhin, Y. Areepong. Efficiency of bivariate copula on the CUSUM chart, The $2^{\text {nd }}$ International Multi Conference of Engineers and Computer Scientist, Hong Kong, 1-4, 2015.

[14] S. Kuvattana, S. Sukparungsee, P. Busbabodhin, Y. Areepong. A comparison of efficiency between multivariate Shewhart and multivariate CUSUM control chart for bivariate copula, The International Conference on Applied Statistics, Pattaya, Thailand, 219-223, 2015.

[15] G. C. Salvadori, C. De Michele, N. T. Kottegoda, R. Rose. Extremes in nature: an approaching using copulas, Water Science and Technology Library, Vol.56, 266, 2007.

[16] F. A. Khoudraji. Contributions a ' l'e tude des copules et a' la mode lisation des valeurs extre`mes bivarie' es', Ph.D. thesis, Universite' Laval, Que' bec, Canada, 1995. 
[17] J. Yan. Enjoy the joy of copulas with a package copula, Journal of Statistical Planning and Inference, Vol.21, No.4, $1-21,2007$

[18] I. Kojadinovic, J. Yan. Modeling Multivariate Distributions with Continuous Margins Using the copula R Package. Journal of Statistical Software, Vol.34, No.9, 1-20, 2010.

[19] V. N. Nyaga, M. Arbyn, M. Aerts. CopulaDTA: An R Package for Copula-Based Bivariate Beta-Binomial Models for Diagnostic Text Accuracy Studies in a Baysian
Framework, Journal of Statistical Software, Vol. 82, 1-26, 2016.

[20] K. F. Vajargah. Comparing Ridge Regression and Principal Components Regression by Monte Carlo Simulation Based on MSE, Journal of Computer Science \& Computational Mathematics, Vol.3, No.2, 25-29, 2013.

[21] J. A. Nelder, R. Mead. A simplex algorithm for function minimization, Computer journal, Vol.7, 308-313, 1965. 